\title{
Behavioral responses of CD-1 mice to conspecific and heterospecific blood odors and to a blood odor component
}

Christian Lahger and Matthias Laska

The self-archived postprint version of this journal article is available at Linköping University Institutional Repository (DiVA):

http:/ / urn.kb.se/ resolve?urn=urn:nbn:se:liu:diva- 144040

N.B.: When citing this work, cite the original publication.

Lahger, C., Laska, M., (2018), Behavioral responses of CD-1 mice to conspecific and heterospecific blood odors and to a blood odor component, Physiology and Behavior, 184, 205-210.

https:// doi.org/ 10.1016/j.physbeh.2017.12.006

Original publication available at:

https:// doi.org/ 10.1016/j.physbeh.2017.12.006

Copyright: Elsevier

http:/ / www.elsevier.com/

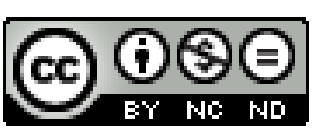


Behavioral responses of CD-1 mice to conspecific and

heterospecific blood odors and to a blood odor component

Christian Lahger and Matthias Laska*

IFM Biology, Linköping University, SE-581 83 Linköping, Sweden

*Correspondence to be sent to:

Matthias Laska, IFM Biology, Linköping University, SE-581 83 Linköping, Sweden email: malas@ifm.liu.se 


\section{Abstract}

The odor of blood may have both aversive and attractive properties for mammals, depending on the species of the odor donor and the species perceiving the odor. To better understand the informational content of blood odor for a prey species we assessed behavioral responses of male CD-1 mice ( $n=60$ ) to the odor of blood of same-sex and opposite-sex conspecifics, of a natural predator of mice (cat), and of a herbivore (horse) and an omnivore (human) nonpredator of mice. Further, we assessed their behavior towards the mammalian blood odor component trans-4,5-epoxy-(E)-2-decenal which recent studies have shown to be as attractive to mammalian predators as the odor of real blood. A two-compartment test arena was used to record approach/avoidance behavior when the animals were presented with an odor in one compartment and a blank control in the other compartment. We found that both conspecific and heterospecific blood odors elicited significant avoidance behavior in the mice whereas a control odor (n-pentyl acetate) did not. The blood odor component trans-4,5-epoxy-(E)-2decenal was also significantly avoided and thus appears to play an important role in the perception of mammalian blood odor in this prey species. These results support the notion that mammalian blood odor contains an olfactory warning signal which elicits an adaptive behavioral avoidance response in a prey species, the mouse. Our finding that the mice avoided the mammalian blood odor component trans-4,5-epoxy-(E)-2-decenal to the same degree as the odor of real blood suggests that this volatile compound might be (part of) this warning signal.

Keywords: blood odor; mouse, avoidance response; habituation; epoxydecenal 


\section{Introduction}

It is well-established that mammalian predators are attracted by the odor of blood and behavioral studies suggest that they may use blood odor to track down wounded prey $[1,2]$. Only few studies, in contrast, assessed behavioral responses of mammalian prey species to blood odor. It seems reasonable to assume that the odor of blood might serve as a warning signal for prey species and thus might elicit adaptive behavioral responses such as increased vigilance, freezing, or flight [3]. This notion is at least partly supported by findings that rats, for example, displayed a clear avoidance of the odor of conspecific blood [4], but did not or only mildly avoid the odor of heterospecific blood from humans or guinea pigs $[5,6]$. Similarly, Mongolian gerbils (Meriones unguiculatus) were found to clearly avoid the odor of blood from stressed conspecifics, but showed only a mild avoidance of the blood odor of nonstressed conspecifics [7], suggesting that blood odor may not only contain a warning signal or "Schreckstoff" [8], but other chemical signals as well. Thus, it seems also reasonable to assume that conspecific blood odor might carry social information, similar to the odor of conspecific urine, faeces, or body odors, and thus might serve as a chemical signal with attractive properties eliciting social or sexual behavior [9]. This notion is in line with the finding that mice displayed less aggressive behavior and directed more sniffing toward mice with conspecific blood smeared on their fur [10].

Chemoanalytical studies have shown that the odor of blood is composed of a complex mixture of volatiles [11], and one component, trans-4,5-epoxy-(E)-2-decenal, has been found to evoke a typical "metallic, blood-like" odor quality in humans [12]. Subsequent behavioral studies reported that mammalian predators such as Siberian tigers (Panthera tigris altaica), African wild dogs (Lycaon pictus), South American bush dogs (Speothos venaticus), and Asian wild dogs (Cuon alpinus) are as attracted to this single volatile compound as they are to the odor of 
real blood [13]. This suggests that the odor of trans-4,5-epoxy-(E)-2-decenal may be perceived by mammalian predators as "blood-like" and thus may act as a "character impact" compound for the odor of blood [14]. This, in turn, raises the question whether this blood odor component may also serve a signaling function for mammalian prey species.

It was therefore the aim of the present study to assess behavioral responses of mice (Mus musculus) to the odor of conspecific blood and to the blood odor component trans-4,5-epoxy(E)-2-decenal. Considering that mice are able to distinguish between urine, fecal, and body odors of male and female conspecifics and thus to detect a chemical signal indicating the sex of the odor donor [15], we decided to assess whether male mice may differ in their response to blood odor from male and female mice, respectively. Further, as rats have been shown to display defensive behavior when exposed to the urine odor of canids and felids, but not to the urine odor of herbivores [16], and thus are able to detect a chemical signal indicating whether a urine odor is from a predator or from a non-predator, we also assessed whether mice may respond differently to blood odor from one of its natural predators, the cat, and from two nonpredator species, the horse and humans.

\section{Materials and methods}

\subsection{Animals}

A total of 60 adult male CD-1 mice were used. The rationale for using this outbred strain of mice was to use animals with a genetic background and a behavioral phenotype which are more similar to that of wild mice than that of inbred strains [17].

The mice were housed individually in standard rodent cages $(40 \times 25 \times 15 \mathrm{~cm})$ and had ad libitum access to food and water. They were kept at a temperature of $22 \pm 1{ }^{\circ} \mathrm{C}$ and under a 
light-dark cycle of 12:12 h (starting at 6:00 a.m. and p.m., respectively). The mice were 90130 days old at the start of testing.

The experiments reported here comply with the Guide for the Care and Use of Laboratory Animals (8 ${ }^{\text {th }}$ edition, The National Academies Press, Washington DC, 2011) and also with current Swedish laws. This study was approved by Linköping's Animal Care and Use Committee (Linköpings djurförsöksetiska nämnd, protocol \#76-12).

\subsection{Odor stimuli}

The mice were presented with eight different odor stimuli:

- Cat blood. We used blood from an adult sterilized male domestic cat (Felis silvestris catus).

- Horse blood. We used blood from an adult male domestic horse (Equus ferus caballus).

- Human blood. We used blood from an adult human male (Homo sapiens).

- Male mouse blood. We used pooled blood from adult male mice (Mus musculus).

- Female mouse blood. We used pooled blood from adult female mice (Mus musculus). Thus, it is possible that animals which were in different phases of their estrous cycle contributed to the corresponding odor stimulus.

The rationale for using these five types of blood was to assess the behavioral responses of mice to the blood odor of a natural predator of mice (cat), of a herbivorous (horse) and an omnivorous (human) non-predator species, and of same-sex (male mouse) and opposite-sex (female mouse) conspecifics.

- Blood odor component. trans-4,5-epoxy-(E)-2-decenal (CAS\# 134454-31-2) is a volatile component found in mammalian blood which evokes a "metallic, blood-like" odor quality in humans [12,18]. This odorant was obtained from AromaLab (Freising, Germany) and presented at a dilution of 1:100 from a stock solution of $5 \mathrm{mg} / \mathrm{mL}$. A previous study has shown that this odorant is as efficient in eliciting behavioral responses in large carnivores as the odor of real blood [13]. Thus, the rationale for using this odorant was to assess whether 
mice, and thus a prey species, display similar behavioral responses to this blood odor component as they do to the odor of real blood.

- Fruity odor. $n$-pentyl acetate (CAS\# 628-63-7) is a volatile component found in a variety of fruits and evokes a "banana-like” odor quality in humans [19]. This odorant was obtained from Sigma-Aldrich (St. Louis, MO) and presented at a dilution of 1:1,000,000. The rationale for using this odorant was to assess the behavioral responses of mice to an odorant that is not found in mammalian blood odor. A previous study has shown that mice are neither attracted nor repelled by this odorant when presented at the concentration used here [20].

- Solvent. Diethyl phthalate (CAS\# 84-66-2) is an organic, near-odorless solvent. This odorant, too, was obtained from Sigma-Aldrich (St. Louis, MO). It was used to dilute the blood odor component and the fruity odor, and it served as the blank stimulus in all tests.

With all types of blood mentioned above, $200 \mu \mathrm{L}$ aliquots were kept at $-20{ }^{\circ} \mathrm{C}$. At the start of the study, all blood samples had been kept frozen for 2-3 months. At the start of each testing day, three aliquots were thawed and warmed up to approximately $22{ }^{\circ} \mathrm{C}$ (room temperature) immediately before they were used. Both the blood odor component and the fruity odor were presented at a concentration that was a factor of 100 above the olfactory detection threshold of mice $[21,22]$.

\subsection{Experimental set-up}

A two-compartment test arena was used [20]. It consisted of a modified standard mouse cage (40 x $25 \times 15 \mathrm{~cm})$ subdivided into two equally sized compartments by a vertical plexiglass wall attached to the lid, with a semi-circular opening at the bottom (diameter $4 \mathrm{~cm}$ ) which allowed a mouse to switch between the compartments. The test arena had a perforated metal floor, and under the floor of each compartment was a petri dish (diameter $9 \mathrm{~cm}$, Sarstedt, Nümbrecht, Germany) with a centrally-placed filter paper (diameter $5.5 \mathrm{~cm}$, Munktell, 
Stockholm, Sweden) impregnated with either $200 \mu \mathrm{L}$ of an odor stimulus or $200 \mu \mathrm{L}$ of the blank stimulus (solvent). Care was taken to place the petri dishes at a position which constituted the center of each of the two compartments of the test arena.

The test arena was placed in a light tent in order to distribute the light evenly and thus to exclude the risk of a side bias due to possible differences in light intensity between the compartments. Additionally, the lights in the testing room were dimmed.

After every 10 -min test trial, the test arena was thoroughly cleaned with $70 \%$ ethanol to minimize the risk of a side bias due to odor contamination. Three test arenas were used in a rotating scheme to allow each one enough time to dry after cleaning.

\subsection{Behavioral test}

Each test trial started by placing a mouse into the test arena, and for 10 minutes the following three parameters were recorded:

- Time spent in each of the two compartments (as an indicator of aversion or preference for a given odor stimulus)

- Number of switches between the two compartments (as an indicator of an animal's overall activity level)

- Number of fecal pellets dropped during the test (as an indicator of an animal's state of fear) [23].

In every 10-min test trial, one side of the two-compartment chamber contained the nearodorless blank stimulus and the other side one of the seven odor stimuli. Two blood odors (or one blood odor and the blood odor component) and the fruity odor $n$-pentyl acetate were tested on the same set of twenty mice. The 60 animals were tested in cohorts of 10 animals. Thus, on any given experimental day, 10 animals were subjected to one 10-min trial each. 
Only when all tests with a given cohort of 10 animals were completed, did we start testing the next cohort of 10 animals.

All mice were tested six times with each of its three designated odor stimuli, three times with a given odorant being presented under the floor of the left compartment and three times under the right. Both the placement of a given odor stimulus under the floor of either the left or the right compartment on a given 10-min test trial and the order of presentation of odors across the 10-min test trials were pseudo-randomized. Similarly, the side of the test arena in which a mouse started a given trial (left or right compartment) was pseudo-randomized and balanced across animals and trials with a given odor stimulus. Only one test trial of ten minutes was performed per mouse and day.

The tests with odor stimuli were preceded by three 10-min trials performed on three consecutive days with the blank stimulus presented on both sides of the test arena to habituate the mice to the test situation and to exclude the possibility of a spontaneous side preference in the animals. During this habituation phase, we recorded the time spent on either side of the arena and found that none of the animals displayed a spontaneous preference for either side. After five mice were tested on a given day, the petri dishes and filter papers were exchanged for new ones to ensure the same odor concentration for each trial. In the case of urination onto the filter paper, the petri dishes were immediately exchanged.

All data were recorded by an observer sitting next to the experimental set-up. Occasional trials during which a second observer was present who was blind as to the placement of the odor stimuli yielded data that differed by not more than $1 \mathrm{sec}$ with regard to the time spent in each of the two sides of the arena, and did not differ at all with regard to the number of switches that an animal performed and the number of fecal pellets that it dropped. Thus, we have no reason to believe that observer bias affected our data collection.

\subsection{Data analysis}


The Wilcoxon signed-rank test was used to assess whether the time spent in an odorized compartment differed from the time spent in the non-odorized (blank) compartment. Possible differences between odor stimuli with regard to the number of switches between compartments, and the number of fecal pellets excreted during odor exposure were first assessed with a Kruskal-Wallis test, followed by pairwise Wilcoxon signed-rank tests with corrections for multiple comparisons. The Spearman rank correlation test was used to assess possible correlations between different parameters across the six 10-min trials performed per odor stimulus and animal. Post-hoc analysis showed that the behavior of the animals did not differ systematically between trials in which they started by being placed in the compartment bearing the odor stimulus or in the compartment bearing the blank stimulus.

As our experimental design only included tests of the type "odor stimulus vs. blank", but not tests of the type “odor stimulus A vs. odor stimulus B”, we assessed so-called absolute preferences or aversions for a given odor stimulus. In order to get a first and indirect indication of whether one of the odor stimuli employed here might elicit stronger behavioral responses than one of the other ones (equivalent to a relative preference or aversion), we also performed a Kruskal-Wallis test, followed by pairwise Wilcoxon signed-rank tests with corrections for multiple comparisons, on the time that the animals spent in the odorized compartment. Post-hoc analysis of our data showed that not all of the data sets were normally distributed. Therefore, data are displayed as median values and their respective median absolute deviation unless otherwise mentioned, and all comparisons between data sets are performed using non-parametric tests.

\section{Results}

\subsection{Approach/avoidance behavior}


All five blood odors and the blood odor component (trans-4,5-epoxy-(E)-2-decenal) elicited significant avoidance behavior. With these six stimuli, the mice spent significantly less time in the odorized compartment compared to the non-odorized (blank) compartment (Wilcoxon signed-rank test, cat blood: $\mathrm{z}=3.071, \mathrm{p}<0.01$; horse blood: $\mathrm{z}=2.806, \mathrm{p}<0.01$; mouse blood (male): $\mathrm{z}=2.144, \mathrm{p}<0.05$; mouse blood (female): $\mathrm{z}=3.977, \mathrm{p}<0.001$; human blood: $\mathrm{z}=4.633$, $\mathrm{p}<0.001$; blood odor component: $\mathrm{z}=2.193, \mathrm{p}<0.05$ ). In contrast, the fruity odor ( $n$-pentyl acetate) was not significantly avoided (fruity odor: $\mathrm{z}=0.453$, $\mathrm{p}>0.05$ ) (Figure 1 ).

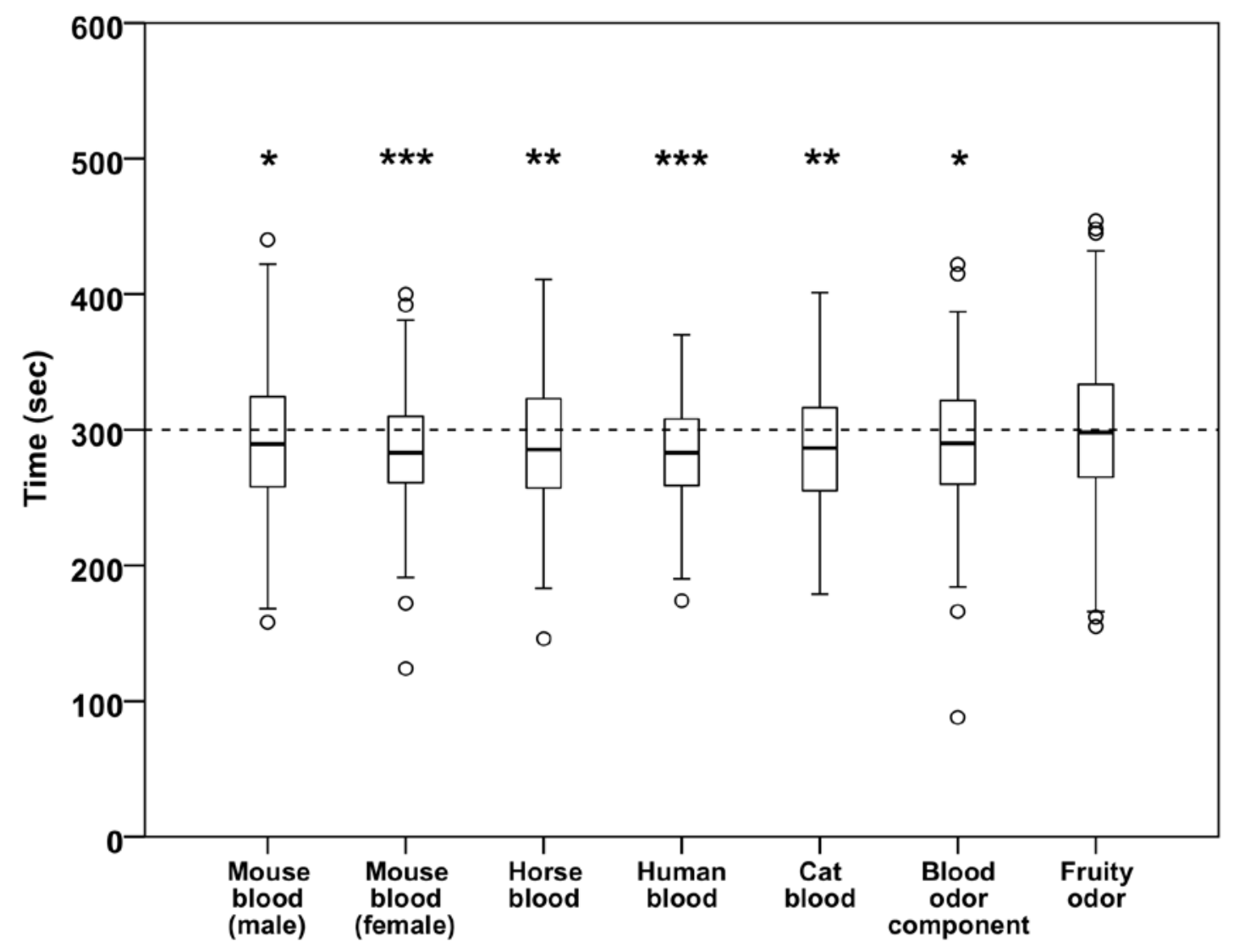

Figure 1 Average time spent in the odorized compartments. The box plots show the median values (bold horizontal line), the 0.25 and 0.75 percentiles (lower and upper box limits), as well as the lowest and highest values (lower and upper whisker ends). Boxplot whiskers are limited to 1.5 times the interquartile ranges. The dotted line represents the halfway point. 
Circles represent outliers. Asterisks indicate a significant deviation from chance (Wilcoxon signed-rank test, $\left.{ }^{*} \mathrm{p}<0.05 ;{ }^{* *} \mathrm{p}<0.01 ; * * * \mathrm{p}<0.001\right)$.

\subsection{Number of switches between compartments}

The median number of switches between the compartments, averaged across the six 10-min trials performed per odor and animal, did not differ significantly between any of the odors (Kruskal-Wallis test, $\mathrm{H}=10.51, \mathrm{p}>0.05$ ) (Figure 2).

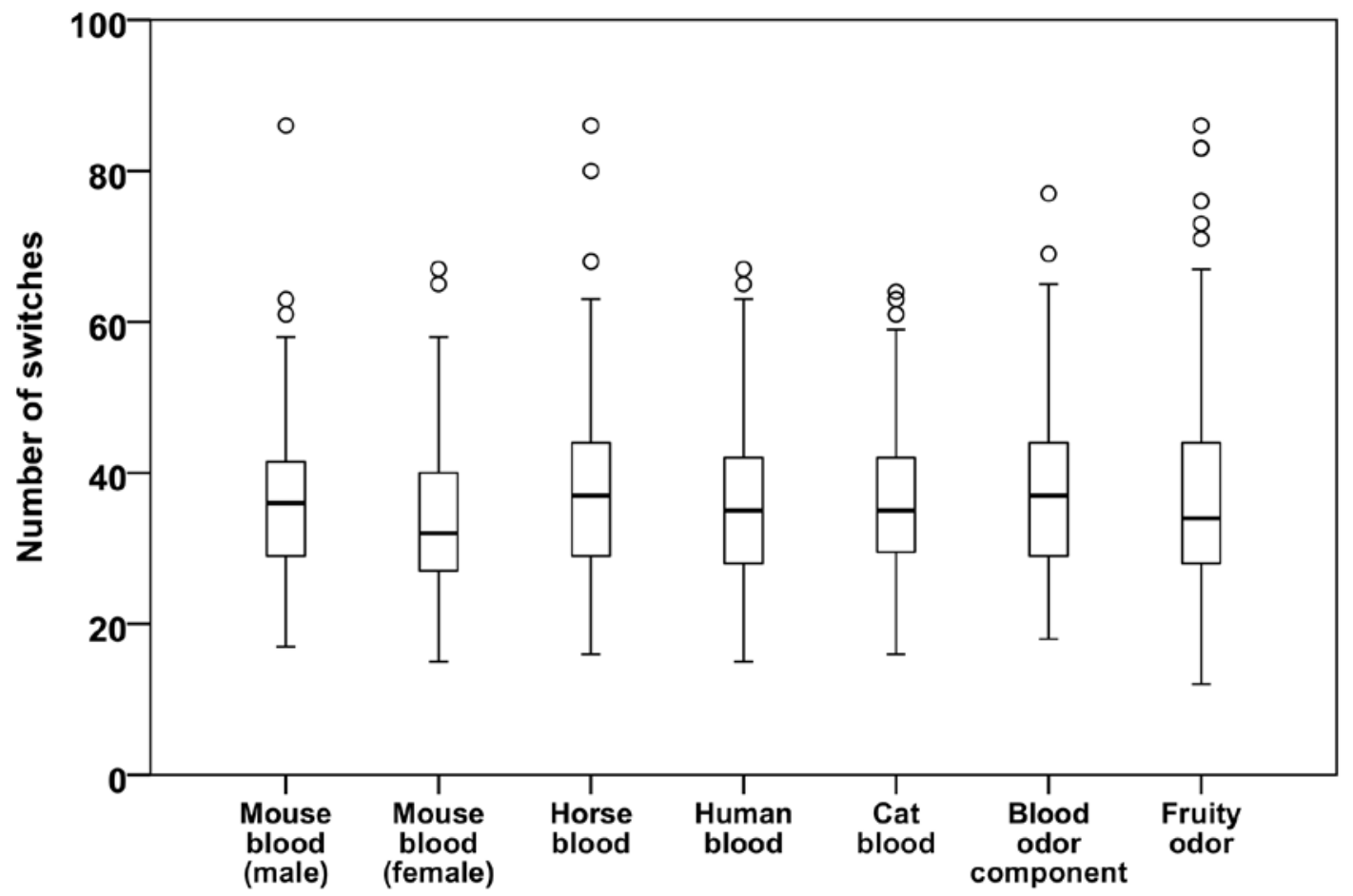

Figure 2 Average number of switches between the compartments. The box plots show the median values (bold horizontal line), the 0.25 and 0.75 percentiles (lower and upper box limits), as well as the lowest and highest values (lower and upper whisker ends). Boxplot whiskers are limited to 1.5 times the interquartile ranges. Circles represent outliers.

\subsection{Number of fecal pellets}


The median number of excreted fecal pellets, averaged across the six 10-min trials performed per odor and animal, did not differ significantly between any of the odors (Kruskal-Wallis test, $\mathrm{H}=10.31, \mathrm{p}>0.05$ ) (Figure 3).

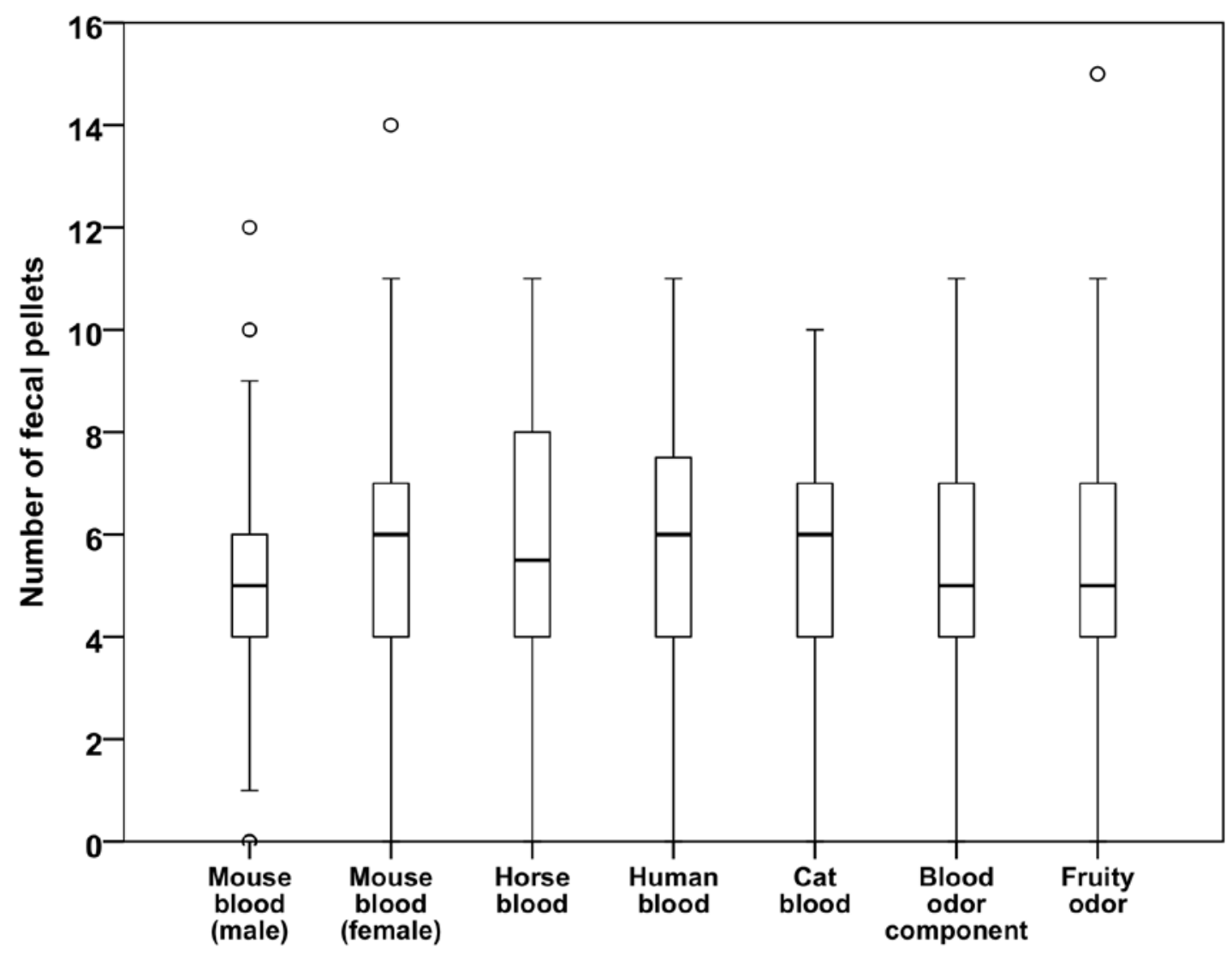

Figure 3 Average number of fecal pellets dropped. The box plots show the median values (bold horizontal line), the 0.25 and 0.75 percentiles (lower and upper box limits), as well as the lowest and highest values (lower and upper whisker ends). Boxplot whiskers are limited to 1.5 times the interquartile ranges. Circles represent outliers.

\subsection{Habituation/sensitization effects across trials}


To assess whether the repeated exposure to an odor led to habituation or sensitization effects, we analyzed whether the animals' behavior changed across the six 10-min trials performed per odor stimulus and animal.

No significant correlation between the time spent in the odorized compartment across the six 10-min trials performed per animal was found with any of the odors (Spearman test, cat blood: $r_{s}=-0.03, p>0.05$; horse blood: $r_{s}=0.08, p>0.05$; mouse blood (male): $r_{s}=0.12, p>0.05$; mouse blood (female): $r_{s}=0.05, p>0.05$; human blood: $r_{s}=-0.02, p>0.05$; blood odor component: $\mathrm{r}_{\mathrm{s}}=-0.09, \mathrm{p}<0.01$; fruity odor: $\mathrm{r}_{\mathrm{s}}=-0.04, \mathrm{p}>0.05$ ) (Figure 4, left panel).

A significant negative correlation between the number of switches between the two compartments across the six 10-min trials performed per animal was found with all of the odors (Spearman test, cat blood: $r_{s}=-0.23, p<0.05$; horse blood: $r_{s}=-0.22, p<0.05$; mouse blood (male): $\mathrm{r}_{\mathrm{s}}=-0.32, \mathrm{p}<0.01$; mouse blood (female): $\mathrm{r}_{\mathrm{s}}=-0.29, \mathrm{p}<0.01$; human blood: $\mathrm{r}_{\mathrm{s}}=-$ 0.30, $\mathrm{p}<0.01$; blood odor component: $\mathrm{r}_{\mathrm{s}}=-0.22, \mathrm{p}<0.05$; fruity odor: $\mathrm{r}_{\mathrm{s}}=-0.32, \mathrm{p}<0.01$ ) (Figure 4, middle panel).

No significant correlation between the number of fecal pellets excreted across the six 10-min trials performed per animal was found with any of the odors (Spearman test, cat blood: $r_{s}=0.08, p>0.05$; horse blood: $r_{s}=0.06, p>0.05$; mouse blood (male): $r_{s}=-0.04, p>0.05$; mouse blood (female): $r_{s}=-0.10, p>0.05$; human blood: $r_{s}=-0.08, p>0.05$; blood odor component: $r_{s}=-$ 0.14, $\mathrm{p}>0.05$; fruity odor: $\mathrm{r}_{\mathrm{s}}=-0.07, \mathrm{p}>0.05$ ) (Figure 4, right panel). 

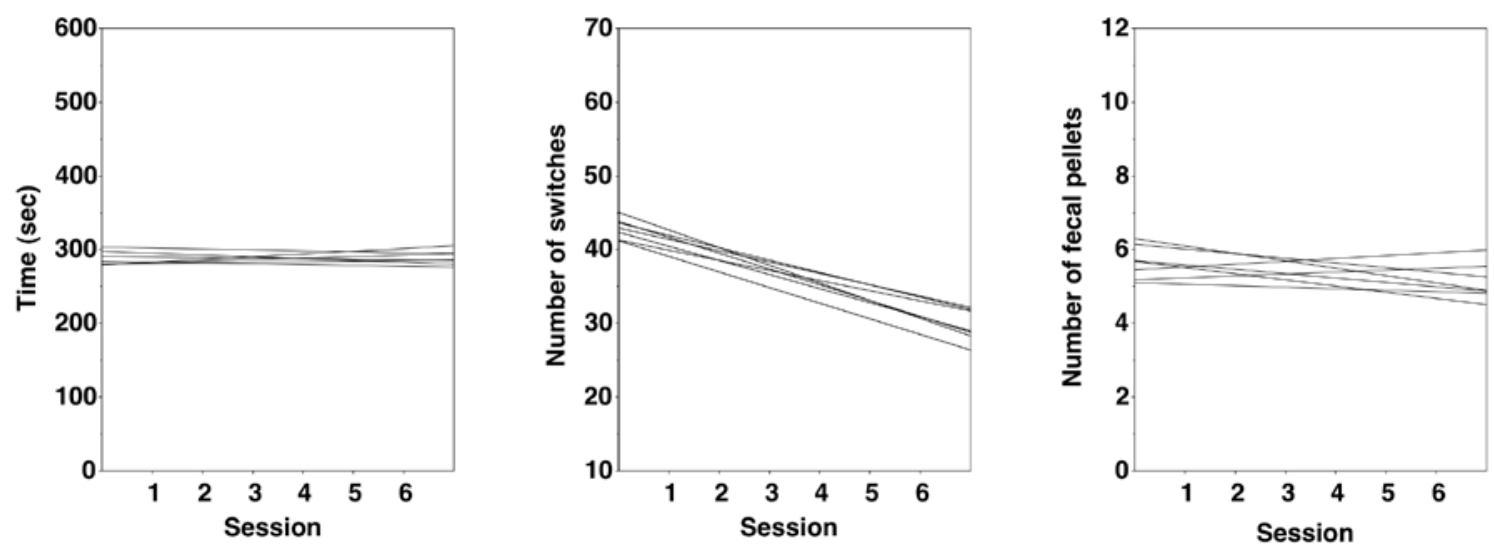

Figure 4 Habituation/sensitization effects across the six 10-min trials performed per animal with regard to the time spent in the odorized compartment (left panel), the number of switches between the two compartments (middle panel), and the number of fecal pellets excreted (right panel). The lines indicate the regression with the best goodness-of-fit according to linear regression analysis for each of the seven odor stimuli.

\subsection{Differences between odors}

In order to assess whether one of the odor stimuli employed here might have elicited stronger behavioral responses than one of the other ones, we compared the animals' approach/avoidance behavior between cat blood, horse blood, male mouse blood, female mouse blood, human blood, and the blood odor component. The time that the mice spent in the odorized compartment did not differ significantly between any of these six blood odors (Kruskal-Wallis test, $\mathrm{H}=3.86$, $\mathrm{p}>0.05$ ).

\section{Discussion}

The results of the present study demonstrate that both conspecific and heterospecific blood odors elicited significant avoidance behavior in the mice. The blood odor component trans4,5-epoxy-(E)-2-decenal was also significantly avoided and thus appears to play an important 
role in the perception of mammalian blood odor in this species. Further, no habituation or sensitization effects with regard to the degree of avoidance or the number of excreted fecal pellets, as an indicator of the animals' state of fear, were found across the six 10-min trials performed per odor stimulus and animal.

\subsection{Informational content of blood odor}

The present results clearly support the notion that mammalian blood odor contains an olfactory warning signal which elicits an adaptive behavioral avoidance response in a prey species, the mouse. Our finding that this warning signal appears to be present in both conspecific and heterospecific blood odor is not trivial considering that the odor of conspecific blood might also contain social signals and therefore might have attractive properties for mice. Based on the present results we cannot decide whether mouse blood odor, similar to the urine, faeces, and body odor of mice [15], may for example also carry information about an odor donor's sex as the male mice tested here avoided both male and female mouse blood odor to the same degree. However, a previous study has shown that mouse blood serum contains protein-bound odorants which, when released via treatment with pronase, enable mice to discriminate between individuals of MHC-congenic conspecifics [24]. This, in turn, suggests that individual and sex-specific odortypes are likely to be present in mouse blood. Our finding that male mice were not attracted by the blood odor of female conspecifics, but rather avoided it as strongly as the blood odor of males, might therefore be interpreted in the way that the olfactory warning signal which is clearly present in mouse blood may take precedence over other, potentially attractive, social signals that are likely to be present at the same time.

Similarly, the results of the present study do not allow us to draw conclusions as to whether mammalian blood odor may contain information about the odor donor being a predator or a non-predator. Several studies provided evidence that prey species such as mice and rats are 
able to discriminate between urine and fecal odors of predators and non-predators, respectively $[3,16]$ and that they respond differently, that is, either with avoidance or with indifference to these two types of odors [25]. Our finding that the mice displayed the same degree of avoidance towards the blood odor of cats, one of their natural predators, and towards the blood odors of humans and horses, two non-predator species, does not exclude the possibility that an olfactory predator signal might be present in cat blood odor as well. Considering that the predator signal which has been found to be present in the odor of urine and faeces of mammalian carnivores is thought to include sulfur compounds that are metabolites of their carnivorous diet [26], and considering that such volatile metabolites are likely to be present in the predator's bloodstream, it seems reasonable to assume that the blood odor of carnivores should also contain this predator signal. This notion is further supported by the finding that mice have been shown to display stronger behavioral responses to the odor of cat faeces when these predators were fed a carnivorous diet compared to when fed a vegetarian diet $[27,28]$. In this context it is also interesting to mention that mice have been shown to lack a fear response when exposed to human urine odor [29] whereas the mice in the present study clearly avoided human blood odor. This finding lends further support to the notion that the warning signal which, based on our findings, is clearly present in mammalian blood odor is not identical with the predator signal that appears to be present in the urine and faeces odor of carnivores [30,31].

\subsection{Behavioral response to the blood odor component}

The mice of the present study did not only significantly avoid the odor of real blood but also the odor of the mammalian blood odor component trans-4,5-epoxy-(E)-2-decenal (see Figure 1). This behavioral response is unlikely to be due to a general avoidance towards novel odors as the mice did not avoid the fruity control odor $n$-pentyl acetate. Rather, this finding supports the notion that mice, similar to humans [18] and mammalian predators [13], might perceive 
this volatile compound as "blood-like" or at least associate it with blood odor. However, whereas mammalian predators such as tigers and wild dogs were found to be attracted by this blood odor component, the mouse, a prey species, avoided it. The only other study so far that assessed behavioral responses of mice to trans-4,5-epoxy-(E)-2-decenal reported that inbred C57BL/6J mice displayed freezing when exposed to this odorant [32]. Although this finding is in line with the present study, it should be mentioned that the method employed by Mori et al [32] included an unphysiologically high concentration of the blood odor component and a test arena lacking an escape room or an alternative compartment which may have contributed to the freezing response which was the only behavioral measure recorded. The present study, in contrast, exposed the mice to a concentration of trans-4,5-epoxy-(E)-2-decenal which was only a factor of 100 above the olfactory detection threshold of mice [22] and thus much closer to the concentration that is found in mammalian blood.

The vast majority of naturally occurring odors is composed of a complex mixture of volatile compounds [14], and the odor of blood is no exception to this rule [11]. Single components of behaviorally relevant odors are usually less effective in eliciting behavioral responses than the naturally occurring mixture that they are normally part of, or are not effective at all [33]. With regard to predator odors, for example, a recent study showed that mice responded to only two out of six sulfur-containing predator odor components with significant avoidance [20]. Even the fox odor component, 2,4,5-trimethylthiazoline (TMT), probably the most widely used odor stimulus in rodent studies assessing fear responses to predator odors, has repeatedly been reported to be less effective compared to the odor of real predator faeces [34,35]. The results of the present study show that the blood odor component trans-4,5-epoxy-(E)-2-decenal does indeed elicit an avoidance response in mice. However, we cannot draw conclusions as to whether this volatile compound may be "the” olfactory warning signal of mammalian blood odor for prey species such as mice, or perhaps may be only one of several blood odor components that elicit avoidance responses that may jointly constitute such a warning signal. 


\subsection{Lack of habituation/sensitization}

The mice of the present study did not systematically vary in their avoidance of the blood odors and the blood odor component across the six 10-min trials performed per stimulus and animal. Similarly, the animals did not show a systematic increase or decrease in the number of fecal pellets that they excreted across trials. Thus, they did not display any habituation or sensitization effects which would suggest a reduction or a potentiation in their avoidance of the blood odors or in their state of fear with repeated exposure to these odors. These findings are in line with several studies which reported that rodents do not habituate in their defensive behavior towards aversive odor stimuli such as predator odors [3,25]. Considering that our mice were laboratory-born and housed individually, and thus had no possibility to encounter any blood odor prior to the study, these findings suggest that the avoidance of mammalian blood odor by a prey species is - similar to the avoidance of predator odors - probably an innate and adaptive behavioral response which is not prone to alterations via learning processes. Our finding of a significant negative correlation between the number of switches between the two compartments across the six 10-min trials performed per animal, and thus of a systematic reduction of the overall activity level of the mice with repeated exposure to all odor stimuli, including the fruity odor, does not contradict this conclusion. Several studies have shown that rats, for example, do not habituate in their avoidance of cat odor with repeated exposure, but resume grooming, feeding, and other social activities after an initial reduction in those behaviors [25,36]. Similarly, mice have been found to sustain their avoidance of predator odor components while reducing their overall activity level across repeated exposures [20]. We attribute the systematic reduction in the overall activity level of the mice across trials that we observed in the present study as well as in a previous study with predator odor components [20] to a continuing familiarization with the test arena and situation. Although our critical tests with odor stimuli were preceded by three 10-min 
familiarization trials without odor stimuli, this might not have been sufficient for the mice to become fully habituated to the test arena and situation. The independence between a sustained avoidance of the blood odors and other behaviors such as motor activity seems adaptive as it would not be useful for a prey animal if behaviors that are necessary in social or foraging contexts were strictly linked with the avoidance response.

Our finding that blood odor and the blood odor component were significantly avoided by the mice (Fig.1) without systematically affecting their overall activity (Fig.2) and the number of fecal pellets they excreted (Fig.3) suggests that an avoidance response to an odor stimulus is not necessarily identical to a fear response. This notion is in line with our finding that the mice hardly ever displayed freezing in response to a blood odor, another behavior thought to be indicative of a fear response.

Taken together, the present results clearly support the notion that both conspecific and heterospecific blood odor contains an olfactory warning signal which elicits an adaptive behavioral avoidance response in a prey species, the mouse. Our finding that the mice avoided the mammalian blood odor component trans-4,5-epoxy-(E)-2-decenal to the same degree as the odor of real blood suggests that this volatile compound might be (part of) this warning signal.

\section{Funding}

This work was supported by institutional funds to ML.

\section{Conflicts of interest}

None.

\section{Acknowledgments}


The animal caretakers at the Center for Biomedical Resources of Linköping University are gratefully acknowledged for their help and support with the animals. Fredrik Södersten from the Division of Pathology at the Swedish University of Agricultural Sciences in Uppsala and Mats Amundin from Kolmården Wildlife Park are gratefully acknowledged for providing cat blood and horse blood, respectively.

\section{References}

[1] M.R. Conover, Predator-prey dynamics: the role of olfaction. Boca Raton, CRC Press, 2007.

[2] D.M. Stoddart, The detection of food, in: D.M. Stoddart (Ed.), The ecology of vertebrate olfaction, Chapman \& Hall, London, 1980, pp. 63-83.

[3] R. Apfelbach, C.D. Blanchard, R.J. Blanchard, R.A. Hayes, I.S. McGregor, The effects of predator odors in mammalian prey species: A review of field and laboratory studies. Neurosci. Biobehav. Rev. 29 (2005) 1123-1144.

[4] D.A. Stevens, N.J. Saplikoski, Rats' reactions to conspecific muscle and blood: evidence for an alarm substance. Behav. Biol. 8 (1973) 75-82.

[5] P. Hornbuckle, T. Beall, Escape reactions to the blood of seleceted mammals by rats. Behav. Biol. 12 (1974) 573-576.

[6] D.A. Stevens, D.A. Gerzog-Thomas, Fright reactions in rats to conspecific tissue. Physiol. Behav. 18 (1977) 47-51.

[7] R. Cocke R, D.D. Thiessen, Chemocommunication among prey and predator species. Anim. Learn. Behav. 14 (1986) 90-92.

[8] K. von Frisch, Über einen Schreckstoff der Fischhaut und seine biologische Bedeutung. Z. Vergl. Physiol. 29 (1942) 46-145. 
[9] T.D. Wyatt, Pheromones and animal behavior, $2^{\text {nd }}$ ed. Cambridge: Cambridge University Press, Cambridge, 2014.

[10] N.K. Sandnabba, The effect of blood signals on aggressive behaviour in mice. Behav. Proc. 41 (1997) 51-56.

[11] M. Kusano, E. Mendez, K.G. Furton, Comparison of the volatile organic compounds from different biological specimens for profiling potential. J. Forensic Sci. 58 (2013) 29-39.

[12] S. Rachamadugu, Characterization of specific volatiles of blood with the potential as predator chemoattractants and as prey warning signals. M.Sc. thesis, Biology, Linköping University (2012).

[13] S. Nilsson, J. Sjöberg, M. Amundin, C. Hartmann, A. Buettner, M. Laska, Behavioral responses to mammalian blood odor and a blood odor component in four species of large carnivores. PLoS ONE. 9 (2014) e112694.

[14] A. Dunkel, M. Steinhaus, M. Kotthoff, B. Nowak, D. Krautwurst, P. Schieberle, T. Hofmann, Nature's chemical signatures in human olfaction. A foodborne perspective for future biotechnology. Angew. Chem. Int. Ed. 53 (2014) 7124-7143.

[15] G.K. Beauchamp, K. Yamazaki, Chemical signalling in mice. Biochem. Soc. Trans. 31 (2003) 147-151.

[16] M. Fendt, Exposure to urine of canids and felids, but not of herbivores, induces defensive behavior in laboratory rats. J. Chem. Ecol. 32 (2006) 2617-2627.

[17] W.E. Crusio, F. Sluyter, R.T. Gerlai, S. Pietropaolo, Behavioral genetics of the mouse, Vol 1. Genetics of behavioral phenotypes. Cambridge, Cambridge University Press, 2013.

[18] A. Buettner, P. Schieberle, Aroma properties of a homologous series of 2,3epoxyalkanals and trans-4,5-epoxyalk-2-enals. J. Agric. Food Chem. 49 (2001) 38813884. 
[19] G. Burdock, Fenaroli's Handbook of Flavor Ingredients, $5^{\text {th }}$ ed., CRC Press, Boca Raton, 2005.

[20] T. Sievert, M. Laska, Behavioral responses of CD-1 mice to six predator odor components. Chem. Senses 41 (2016) 399-406.

[21] J.C. Walker, R.J. O’Connell, Computerized odor psychophysical testing in mice. Chem. Senses 11 (1986) 439-453.

[22] A. Sarrafchi, M. Laska, Olfactory sensitivity for the mammalian blood odor component trans-4,5-epoxy-(E)-2-decenal in CD-1 mice. Perception 46 (2017) 333-342.

[23] K.E. Bailey, J.N. Crawley, Anxiety-related behaviors in mice. in: J.J. Buccafusco (Ed.), Methods of behavior analysis in neuroscience, $2^{\text {nd }}$ ed., Boca Raton, CRC Press, 2009, pp. 77-101.

[24] K. Yamazaki, G.K. Beauchamp, A. Singer, J. Bard, E.A. Boyse, Odortypes: their origin and composition. PNAS. 96 (1999) 1522-1525.

[25] R.A. Dielenberg, I.S. McGregor, Defensive behavior in rats toward predatory odors: a review. Neurosci. Biobehav. Rev. 25 (1999) 597-609.

[26] D.L. Nolte, R. Mason, G. Epple, E. Aronov, D.L. Campbell, Why are predator urines aversive to prey? J. Chem. Ecol. 20 (1994) 1505-1516.

[27] F. Berton, E.W. Vogel, C. Belzung, Modulation of mice anxiety in response to cat odor as a consequence of predators diet. Physiol. Behav. 65 (1998) 247-254.

[28] A.E. Scherer, D.L. Smee, A review of predator diet effects on prey defensive responses. Chemoecology 26 (2016) 83-100.

[29] G.F. Rivard, E.G. Moser, S.P. D’Ambrose, D.M. Lin, Lack of fear response in mice (Mus musculus) exposed to human urine odor. J. Amer. Soc. Lab. Anim. Sci. 53 (2014) 141-145. 
[30] L.E. Belton, N. Ball, J.M. Waterman, P.W. Bateman, 2007. Do Cape ground squirrels (Xerus inauris) discriminate between olfactory cues in the faeces of predators versus non-predators? Afr. Zool. 42 (2007) 135-138.

[31] D.T. Blumstein, L. Barrow, M. Luterra, Olfactory predator discrimination in yellowbellied marmots. Ethology 114 (2008) 1135-1143.

[32] K. Mori, K. Osada, M. Amaike, Mammalian blood odorant and chirality: synthesis and sensory evaluation by humans and mice of the racemate and enantiomers of trans-4,5epoxy-(E)-2-decenal. Tetrahedron-Asymmetry 26 (2015) 861-867.

[33] O. Nevo, R. Orts Garri, L.T. Hernandez Salazar, S. Schulz, E.W. Heymann, M. Ayasse, M. Laska, Chemical recognition of fruit ripeness in spider monkeys (Ateles geoffroyi). Sci. Rep. 5 (2015) 14895.

[34] I.S. McGregor, L. Schrama, P. Ambermoon, R.A. Dielenberg, Not all ”predator odours” are equal: cat odour but 2,4,5-trimethylthiazoline (TMT: fox odour) elicits specific defensive behaviours in rats. Behav. Brain Res. 129 (2002) 1-16.

[35] R. Apfelbach, M.H. Parsons, H.A. Soini, M.V. Novotny, Are single odorous components of a predator sufficient to elicit defensive behaviors in prey species? Front. Neurosci. 9 (2015) 263.

[36] L.G. Staples, Predator odor avoidance as a rodent model of anxiety: learning-mediated consequences beyond the initial exposure. Neurobiol. Learn. Mem. 94 (2010) 435-445. 